\title{
ON A CLASS OF MARKOV CHAINS
}

\author{
A. G. PAKES \\ (Received 30 September 1968; revised 1 April 1969) \\ Communicated by P. D. Finch
}

\section{Introduction}

Until recently, very little work has been done on the second order properties of Markov chains. Craven [1] has studied the joint distributions of Markov chains having a Borel subset of $n$-dimensional Euclidean space as state space. His idea was to consider the process as a time series.

More recently, Daley [2] has introduced a class of Markov chains on a Borel subset of the real line, which he calls stochastically monotone Markov chains. Under certain conditions, he has shown that for stationary Markov chains, $\left\{X_{n}\right\}_{0}^{\infty}$ of this class, the correlation coefficient of $f\left(X_{0}\right)$ and $f\left(X_{n}\right)$ is a monotone non-increasing sequence, where $f(\cdot)$ is a monotone function from the state space of $\left\{X_{n}\right\}$ into the real line. In a further study, Daley [3] has considered a special Markov chain of this class, namely the imbedded waiting time Markov chain in the stationary $G I / G / 1$ queue. In this particular case, the proofs on the behaviour of the sequence of correlation coefficients are simpler in detail than they are for the more general case.

In this paper, we shall only be concerned with Markov chains on the nonnegative integers. In Section 2, we introduce the required notation and two key results which will be used in discussing the second order properties. Section 3 presents some simple properties of stochastically monotone Markov chains on the non-negative integers, and in Section 4 we discuss their second order properties. We show that the type of arguments used in [3] carry over to the present case, and we consider a somewhat wider class than that of stationary Markov chains.

\section{General considerations}

Let $\left\{X_{n}\right\}_{0}^{\infty}$ be a Markov chain with the non-negative integers as state space, $\mathscr{X}$. Let $p_{i j}^{(n)}$ be the $n$-step transition probability from state $i$ to $j$, and let $p_{i j} \equiv p_{i j}^{(1)}$. Finally let $f(\cdot): \mathscr{X} \rightarrow B$, where $B$ is a countable subset of the real line. We shall always assume that $E\left\{f\left(X_{0}\right)\right\}$ is finite, that is, the series $\sum_{i=0}^{\infty} a_{i} f(i)$ converges absolutely, where $a_{i}=\operatorname{Pr}\left\{X_{0}=i\right\}$. 
Define $\theta_{j}^{(n)}$ for $n=0,1, \cdots$ and $j \in \mathscr{X}$ by

$$
\theta_{j}^{(n)}=\sum_{i=0}^{\infty} f(i) \operatorname{Pr}\left\{X_{0}=i, X_{n}=j\right\}=E\left\{f\left(X_{0}\right) ; X_{n}=j\right\}
$$

Then when the Markov chain is ergodic and $\operatorname{Pr}(\cdot)$ is the stationary probability measure for the process $\left\{X_{n}\right\}$,

$$
\theta_{j}^{(n)}=\sum_{i=0}^{\infty} f(i) \operatorname{Pr}\left\{X_{m}=i, X_{n+m}=j\right\} \quad(m=0,1, \cdots)
$$

Defining for $n, j=0,1, \cdots$,

$$
\phi_{j}^{(n)}=\sum_{i=0}^{j} \theta_{j}^{(n)}=\sum_{i=0}^{\infty} f(i) \operatorname{Pr}\left\{X_{0}=i, X_{n} \leqq j\right\}=E\left\{f\left(X_{0}\right) ; X_{n} \leqq j\right\}
$$

it follows from the absolute convergence of $\sum_{i=0}^{\infty} f(i) \operatorname{Pr}\left\{X_{0}=i\right\}$ and the dominated convergence theorem that

$$
\phi^{(n)}=\lim _{j \rightarrow \infty} \phi_{j}^{(n)}=\sum_{i=0}^{\infty} \theta_{i}^{(n)}=E\left\{f\left(X_{0}\right)\right\}
$$

It follows by absolute convergence again and the Markovian nature of $\left\{X_{n}\right\}$ that

$$
\theta_{j}^{(n)}=\sum_{i=0}^{\infty} \theta_{i}^{(n-1)} p_{i j}=\sum_{i=0}^{\infty} \theta_{i}^{(0)} p_{i j}^{(n)} \quad(n=1,2, \cdots) .
$$

When the chain is ergodic, the absolute convergence of $\sum_{i=0}^{\infty} \theta_{i}^{(0)}$ and the dominated convergence theorem imply that $\theta_{j}^{(n)} \rightarrow \pi_{j} E\left\{f\left(X_{0}\right)\right\}(n \rightarrow \infty)$, where $\left\{\pi_{j}\right\}_{0}^{\infty}$ is the limiting distribution of $\left\{X_{n}\right\}$.

Suppose that $\left\{X_{n}\right\}$ is irreducible and aperiodic, that the $\theta_{j}^{(n)}$ are non-negative for all $n$ and $j$, that $\lim _{n \rightarrow \infty} \theta_{j}^{(n)}=\theta_{j}$ exists $(j=0,1, \cdots)$ and $\theta_{j}$ is positive for some particular $j$, and that $\sum_{j=0}^{\infty} \theta_{j}^{(0)}$ is finite. Then it is easily seen that $\left\{X_{n}\right\}$ is positive recurrent, that $\theta_{j}$ is positive for all $j$ and that $\sum_{j=0}^{\infty} \theta_{j}=\sum_{j=0}^{\infty} \theta_{j}^{(n)}(n=0,1$, $\cdots)$. Theorem 1 shows that the same conclusions follow if we assume the finiteness of $\sum_{j=0}^{\infty} \theta_{j}$ rather than that of $\sum_{j=0}^{\infty} \theta_{j}^{(0)}$.

THEOREM 1. Let $\left\{X_{n}\right\}$ be an irreducible aperiodic Markov chain on a countable state space I and let $\left\{\lambda_{j}^{(0)}\right\}$ be a non-negative measure on I. Define $\lambda_{j}^{(n)}=\sum_{i \in I} \lambda_{i}^{(0)} p_{i j}^{(n)}$ (possibly $=\infty$ ), and suppose that $\lim _{n \rightarrow \infty} \lambda_{j}^{(n)}=\lambda_{j}$ exists for each $j$, that $\lambda_{j}>0$ for some particular $j$, and that $\sum_{j \in I} \lambda_{j}<\infty$. Then the Markov chain $X_{n}$ is positive recurrent, every $\lambda_{j}>0$, and $\sum_{j \in I} \lambda_{j}^{(n)}=\sum_{j \in I} \lambda_{j}$ for all $n$.

Proof. Fatou's lemma applied to $\lambda_{j}^{(n+m)}=\sum_{i \in I} \lambda_{i}^{(m)} p_{i j}^{(n)}$ implies that

$$
\lambda_{j}=\liminf _{m \rightarrow \infty} \lambda_{j}^{(m+n)} \geqq \sum_{i \in I} \liminf _{m \rightarrow \infty} \lambda_{i}^{(m)} p_{i j}^{(n)}=\sum_{i \in I} \lambda_{i} p_{i j}^{(n)} .
$$

That $\lambda_{j} \geqq 0$ and that $\sum_{j \in I} \lambda_{j}$ is finite imply that we have equality in the last expression and so by a theorem of Foster [4], the chain is positive recurrent and $\lambda_{j}>0$ for each $j$. 
Now let $\lambda_{j}^{(0)}(N)=\lambda_{j}^{(0)}$ if $j \in I_{N}$ and $\lambda_{j}^{(0)}(N)=0$ otherwise, where $\left\{I_{N}\right\}$ is a monotone increasing sequence of finite subsets of $I$ for which $I_{N} \rightarrow I(N \rightarrow \infty)$. Then $\infty>\sum_{j \in I} \lambda_{j}^{(0)}(N) \rightarrow \sum_{j \in I} \lambda_{i}^{(0)}$, the convergence being monotone from below. With the definition of $\lambda_{j}^{(n)}(N)=\sum_{i \in I} \lambda_{i}^{(0)}(N) p_{i j}^{(n)}, \lambda_{j}^{(0)} \geqq 0$ implies the monotone convergence of $\lambda_{j}^{(n)}(N)$ to $\lambda_{j}^{(n)}(N \rightarrow \infty)$ for every $n$ and $j$. Tonelli's theorem shows that $\sum_{j \in I} \lambda_{j}^{(0)}=\sum_{j \in I} \lambda_{j}^{(n)}(n=0,1, \cdots)$ and the chain being ergodic implies, by the dominated convergence theorem, the existence of $\lambda_{j}(N)=\lim _{n \rightarrow \infty} \lambda_{j}^{(n)}(N)=$ $\pi_{j} \sum_{i \in I} \lambda_{i}^{(0)}(N)$, where $\left\{\pi_{j}\right\}$ is the limiting distribution of $\left\{X_{n}\right\}$. This and monotone convergence imply that $\sum_{j \in I} \lambda_{j}=\lim _{N \rightarrow \infty} \sum_{j \in I} \lambda_{j}(N)=\lim _{N \rightarrow \infty} \sum_{i \in I} \lambda_{i}^{(0)}(N)=$ $\sum_{i \in I} \lambda_{i}^{(0)}$ and the proof is complete.

Let $g(\cdot): \mathscr{X} \rightarrow C$, where $C$ is a countable subset of the real line. An object of this paper is the study of the second order moments $E\left\{f\left(X_{0}\right) g\left(X_{n}\right)\right\}=M^{(n)}$ of the Markov chain $\left\{X_{n}\right\}$ with certain initial distributions. By the CauchySchwartz inequality, these moments are well defined if both $E\left\{f^{2}\left(X_{0}\right)\right\}$ and $E\left\{g^{2}\left(X_{n}\right)\right\}$ are finite. If $f(\cdot)$ is a non-negative (or non-positive) function and if $0<E\left\{f\left(X_{0}\right)\right\}<\infty$, then, as Craven [1] has shown, these second order moments can be expressed in terms of the first order moments of a related Markov chain which is in general non-stationary. Specifically, let $\left\{Y_{n}\right\}_{0}^{\infty}$ be the Markov chain whose $n$-step transition probabilities are identical to those of $\left\{X_{n}\right\}$ but whose initial distribution is given by $\theta_{j}^{(0)} /\left[E\left\{f\left(X_{0}\right)\right\}\right]$. Then $M^{(n)}=E\left[f\left(X_{0}\right)\right\} E\left\{g\left(Y_{n}\right)\right\}$.

In discussing these second order moments, we use the following theorem.

THEOREM 2. Let $X$ and $Y$ be (not necessarily independent) random variables such that $\operatorname{Pr}\{Y \leqq x\} \geqq \operatorname{Pr}\{X \leqq x\}$ (all real $x$ ), and let $f(x)$ be a monotonic non-decreasing function with $E\{|f(X)|\}<\infty$. Then if $f(\cdot)$ is non-negative or if $X$ and $Y$ have the same distribution,

$$
E\{f(X) ; Y \leqq x\}-E\{f(X) ; X \leqq x\} \geqq 0 \quad \text { (all } x) .
$$

Proof. The left hand side of the last inequality is equal to

$$
\begin{aligned}
E\{f(X) ; X & >x, Y \leqq x\}-E\{f(X) ; X \leqq x, Y>x\} \\
& \geqq E\{f(x) ; X>x, Y \leqq x\}-E\{f(x) ; X \leqq x, Y>x\} \\
& =f(x)[\operatorname{Pr}\{Y \leqq x\}-\operatorname{Pr}\{X \leqq x\}] \geqq 0 .
\end{aligned}
$$

We shall make frequent use of the following result.

LEMMA 1. Let $\left\{a_{j}\right\}$ and $\left\{b_{j}\right\}(j=0,1, \cdots)$ be two sequences such that $\sum_{j=0}^{\infty} a_{j} b_{j}$ is convergent. Let $A_{j}$ be the $j$-th partial sum of the series $\sum_{j=0}^{\infty} a_{j}$, which is supposed convergent with sum $A$. If $\left\{b_{j}\right\}$ is a bounded sequence, then

$$
\sum_{j=0}^{\infty} a_{j} b_{j}=\sum_{j=0}^{\infty}\left[A-A_{j}\right]\left[b_{j+1}-b_{j}\right]+b_{0}
$$

and if $\left\{b_{j}\right\}$ has a finite limit $b$, then 


$$
\sum_{j=0}^{\infty} a_{j} b_{j}=\sum_{j=0}^{\infty} A_{j}\left[b_{j}-b_{j+1}\right]+A b .
$$

The first equality holds if $\sum_{j=0}^{\infty} a_{j} b_{j}$ converges absolutely and if $\left\{b_{j}\right\}$ is a monotone sequence.

Proof. Abel's transformation shows that

$$
\begin{aligned}
\sum_{j=0}^{\infty} a_{j} b_{j} & =\lim _{M \rightarrow \infty}\left\{\sum_{j=0}^{M-1} A_{j}\left[b_{j}-b_{j+1}\right]+A_{M} b_{M}\right\} \\
& =\lim _{M \rightarrow \infty}\left\{\sum_{j=0}^{M-1}\left(A-A_{j}\right)\left(b_{j+1}-b_{j}\right)-\left(A-A_{M}\right) b_{M}+b_{0}\right\}
\end{aligned}
$$

The second result follows immediately, and the first follows on noting that under either set of hypotheses, $\left(A-A_{M}\right) b_{M} \rightarrow 0(M \rightarrow \infty)$.

\section{Stochastically monotone Markov chains}

Let $Q_{i j}=\sum_{k=0}^{j} p_{i k}$ and $Q_{i j}^{(n)}=\sum_{k=0}^{j} p_{i k}^{(n)}(n=2,3, \cdots)$. Let $P$ be the matrix $\left[p_{i j}\right]$ and $P^{n}=\left[p_{i j}^{(n)}\right]$.

Definition. A Markov chain $\left\{X_{n}\right\}_{0}^{\infty}$ having one step transition probabilities $p_{i j}$ is said to be stochastically monotone if for each $j=0,1, \cdots, Q_{i j} \geqq Q_{i+1, j}$ $(i=0,1, \cdots)$.

If this condition holds, we will also say that $P$ is stochastically monotone. This definition is the specialization to Markov chains on the non-negative integers of Daley's [2] original definition, which applied to Markov chains on a Borel subset of the real line. Daley has shown that if $P$ is stochastically monotone, then so is $P^{n}$. The remainder of this section is devoted to the derivation of some simple properties of stochastically monotone Markov chains.

LeMma 2. If $P$ is stochastically monotone, then $\left\{Q_{0 j}^{(n)}\right\}_{n=0}^{\infty}$ is monotone nonincreasing for each $j$, and in particular $\left\{p_{00}^{(n)}\right\}$ is a monotone non-increasing renewal sequence.

PRoOF. $Q_{0 j}^{(n+1)}=\sum_{i=0}^{\infty} p_{0 i} Q_{i j}^{(n)} \leqq Q_{0 j}^{(n)} \sum_{i=0}^{\infty} p_{0 i}$, the inequality following from the stochastic monotonicity of $P^{n}$.

COROLLARY 1. Let $\left\{X_{n}\right\}$ be an irreducible stochastically monotone Markov chain. Define $P^{(n)}(x)=\sum_{j=0}^{\infty} p_{0 j}^{(n)} x^{j}(0 \leqq x \leqq 1)$, then $P^{(n)}(x) \downarrow \Pi(x)(n \rightarrow \infty)$ where $\Pi(x)$ is the generating function of the limiting distribution.

Proof. Daley [2] has shown that an irreducible stochastically monotone chain is aperiodic, and so the limiting distribution exists; being defective if the Markov chain is not positive recurrent. For $x=1$, there is nothing to prove, while for $0 \leqq x<1$, the result follows from 
by Lemma 2 .

$$
1 \geqq P^{(n)}(x)=(1-x) \sum_{j=0}^{\infty} Q_{0 j}^{(n)} x^{j} \geqq P^{(n+1)}(x) \geqq 0
$$

Corollary 2. Let $f(\cdot): \mathscr{X} \rightarrow B$, a countable subset of the real line, be a nondecreasing function such that $E\left\{\left|f\left(X_{n}\right)\right|\right\}<\infty$ for any initial distribution which may be used. Define the following conditional expectations: $b_{i}^{(n)}=E\left\{f\left(X_{n}\right) \mid X_{0}=i\right\}=$ $\sum_{j=0}^{\infty} p_{i j}^{(n)} f(j)$ and $b^{(n)}(\mathscr{A})=\sum_{i=0}^{\infty} a_{i} b_{i}^{(n)}$ where $\mathscr{A}=\left\{a_{i}\right\}_{0}^{\infty}$ is an arbitrary initial distribution. Then $\left\{b_{0}^{(n)}\right\}_{n=0}^{\infty}$ is a non-decreasing sequence, $\left\{b_{i}^{(n)}\right\}_{i=0}^{\infty}$ is a non-decreasing sequence for each fixed $n$, and $b_{0}^{(n)} \leqq b^{(n+m)}(\mathscr{A})$ for each $n, m=0,1, \cdots$ and any $\mathscr{A}$.

Proof. We have $E\left\{\left|f\left(X_{n}\right)\right| \mid X_{0}=i\right\}=m_{i} \leqq K$ for some finite constant $K$. For if not, then there exists a strictly increasing sequence $i_{1}, i_{2}, \cdots, i_{k}, \cdots$ of positive integers such that $m_{i_{1}} \geqq 1, \cdots, m_{i_{k}} \geqq k, \cdots$, and then setting $a_{i_{k}}=6 / \pi^{2} k^{2}$, $a_{i}=0$ if $i \neq i_{k}$ for some $k$, we have $\sum a_{i}=1, \sum a_{i} m_{i} \geqq 6 \pi^{-2} \sum 1 / k=\infty$. Thus the existence of all quantities is guaranteed. On applying Lemma 1 to the defining series for $b_{i}^{(n)}$, we have

$$
b_{i}^{(n)}=f(0)+\sum_{j=0}^{\infty}[f(j+1)-f(j)]\left[1-Q_{i j}^{(n)}\right] .
$$

The conclusions follow on noting that the stochastic monotonicity of $P^{n}$ and Lemma 2 imply that $Q_{0 j}^{(n)} \geqq Q_{0 j}^{(n+m)} \geqq Q_{i j}^{(n+m)}(n, m, i, j=0,1, \cdots$.

We can derive upper and lower bounds for the state probabilities of a stochastically monotone Markov chain.

LEMMA 3. Let $\left\{X_{n}\right\}$ be a stochastically monotone Markov chain with an arbitrary initial distribution and let $\operatorname{Pr}(\cdot)$ be the probability measure so defined. For a fixed $n$ define $h$ by $h=\min \left\{i \mid \operatorname{Pr}\left\{X_{n}=i\right\} \neq 0\right\}$. Then $\operatorname{Pr}\left\{X_{n+1} \leqq j\right\} \leqq Q_{h j}$, and if $j \geqq h$, $\operatorname{Pr}\left\{X_{n+1} \leqq j\right\} \geqq Q_{j j} \operatorname{Pr}\left\{X_{n} \leqq j\right\}$.

Proof. By the definition of $h$, we have $\operatorname{Pr}\left\{X_{n+1} \leqq j\right\}=\sum_{i=h}^{\infty} \operatorname{Pr}\left\{X_{n}=i\right\} Q_{i j}$. The first part of the lemma follows easily. The second part follows because $\operatorname{Pr}\left\{X_{n+1} \leqq j\right\} \geqq \sum_{i=h}^{j} \operatorname{Pr}\left\{X_{n}=i\right\} Q_{i j} \geqq Q_{j j} \sum_{i=h}^{j} \operatorname{Pr}\left\{X_{n}=i\right\}$.

\section{Second order properties}

THEOREM 3. Let $\left\{X_{n}\right\}$ be a stochastically monotone Markov chain with an initial distribution such that $\operatorname{Pr}\left\{X_{1} \leqq j\right\} \geqq \operatorname{Pr}\left\{X_{0} \leqq j\right\}(j=0,1, \cdots)$. Let $f(\cdot)$ be a non-decreasing non-negative function from $\mathscr{X}$ to a countable subset of the real line such that $E\left\{f\left(X_{0}\right)\right\}<\infty$; or let $f(\cdot)$ be simply non-decreasing and such that $E\left\{\left|f\left(X_{0}\right)\right|\right\}<\infty$ and let $X_{0}$ and $X_{1}$ be identically distributed. Then for each fixed $j=0,1, \cdots, \phi_{j}^{(n+1)} \geqq \phi_{j}^{(n)}(n=0,1, \cdots)$. 
Proof. The hypotheses of Theorem 2 are satisfied, so we have $\phi_{j}^{(1)} \geqq \phi_{j}^{(0)}$ $(j=0,1, \cdots)$. Since $\phi_{j}^{(n)}=\sum_{i=0}^{\infty} \theta_{i}^{(n-1)} Q_{i j}$, we have from Lemma 1 that

$$
\phi_{j}^{(n+1)}-\phi_{j}^{(n)}=\sum_{i=0}^{\infty}\left[\phi_{i}^{(n)}-\phi_{i}^{(n-1)}\right]\left[Q_{i j}-Q_{i+1, j}\right]
$$

The stochastic monotone property implies the existence of $\lim _{i \rightarrow \infty} Q_{i j}$. The theorem is now proved by using the stochastic monotone property and arguing inductively.

If the Markov chain in Theorem 3 is irreducible, then, by a theorem of Daley [2], it is aperiodic, and Fosters theorem [4] shows that if $X_{0}$ and $X_{1}$ are identically distributed, the chain is positive recurrent and the initial distribution is then the limiting distribution. If the first set of hypotheses holds and the chain is irreducible, then it is ergodic. For we can choose a function $f(\cdot)$ such that $\phi_{j}^{(0)}>0$ for some particular $j$, and so we have $0<\phi_{j}^{(0)} \leqq \cdots \leqq \phi_{j}^{(n)} \leqq \phi_{j}^{(n+1)} \leqq \cdots$. If the chain is not ergodic, then $\lim _{n \rightarrow \infty} Q_{i j}^{(n)}=0(i, j=0,1, \cdots)$ and the dominated convergence theorem then shows that $\lim _{n \rightarrow \infty} \phi_{j}^{(n)}=0$, a contradiction.

If $f(i)$ is the unit step function having its jump at $k$, then under the conditions of Theorem $3, \operatorname{Pr}\left\{X_{0} \geqq k, X_{n} \leqq j\right\} \leqq \operatorname{Pr}\left\{X_{0} \geqq k, X_{n+1} \leqq j\right\}(n, j, k=0,1, \cdots)$. Lemma 1 then shows that $E\left\{g\left(X_{n}\right) ; X_{0} \geqq k\right\} \geqq E\left\{g\left(X_{n+1}\right) ; X_{0} \geqq k\right\}$ provided these expectations exist and $g(\cdot)$ is a non-decreasing function from $\mathscr{X}$ to a countable subset of the real line.

Theorem 3 covers the special case of stationary Markov chains. A version of Theorem 3 for stationary Markov chains on a Borel subset of the real line is implicitly contained in the proof of Theorem 4 of [2].

We now turn our attention to the second order moments $M^{(n)}=E\left\{f\left(X_{0}\right)\right.$ $\left.g\left(X_{n}\right)\right\}$ where $f(\cdot)$ and $g(\cdot)$ are functions from $\mathscr{X}$ to countable subsets of the real line. We assume that $f(\cdot), g(\cdot)$ and the initial distribution of $\left\{X_{n}\right\}$ is such that both $E\left\{f^{2}\left(X_{0}\right)\right\}$ and $E\left\{f^{2}\left(X_{n}\right)\right\}$ are finite, so that by the Cauchy-Schwartz inequality, the $M^{(n)}$ are well defined.

THEOREM 4. Let $\left\{X_{n}\right\}$ be a stochastically monotone Markov chain with an initial distribution such that $\operatorname{Pr}\left\{X_{1} \leqq j\right\} \geqq \operatorname{Pr}\left\{X_{0} \leqq \mathrm{j}\right\}(j=0,1, \cdots)$. Let $f(\cdot)$ and $g(\cdot)$ be monotone non-negative functions from $\mathscr{X}$ to countable subsets of the real line; or let $X_{0}$ and $X_{1}$ be identically distributed and let $f(\cdot)$ and $g(\cdot)$ be simply monotone functions. In either case let both $E\left\{f^{2}\left(X_{0}\right)\right\}$ and $E\left\{g^{2}\left(X_{n}\right)\right\}$ be finite. Then $\left\{M^{(n)}\right\}_{0}^{\infty}$ is a monotone non-increasing sequence if both $f(\cdot)$ and $g(\cdot)$ are both monotone nondecreasing or non-increasing, and it is a monotone non-decreasing sequence if one of $f(\cdot)$ and $g(\cdot)$ is monotone non-decreasing and the other is non-increasing. If $\left\{X_{n}\right\}$ is irreducible, then $\lim _{n \rightarrow \infty} M^{(n)}=E\left\{f\left(X_{0}\right)\right\} \sum_{j=0}^{\infty} g(j) \pi_{j}$, where $\left\{\pi_{i}\right\}$ is the limiting distribution.

If $\left\{X_{n}\right\}$ is irreducible and stationary, then $\gamma^{(n)}=\operatorname{cov}\left\{f\left(X_{0}\right), g\left(X_{n}\right)\right\}$ converges monotonically to zero from above or below, according as $f(\cdot)$ and $g(\cdot)$ have the same monotone behaviour or not. 
Proof. It clearly suffices to consider the case where both $f(\cdot)$ and $g(\cdot)$ are monotone non-decreasing. It is easily seen that $M^{(n)}=\sum_{j=0}^{\infty} \theta_{j}^{(n)} g(j)$, and the hypotheses together with the Cauchy-Schwartz inequality show that the series converges absolutely and so Lemma 1 shows that

$$
M^{(n)}=g(0) \phi^{(n)}+\sum_{j=0}^{\infty}\left[\phi^{(n)}-\phi_{j}^{(n)}\right][g(j+1)-g(j)] \quad(n=0,1, \cdots)
$$

and the first conclusion follows on using Theorem 3 . Using the monotone convergence theorem in the last expression, the remarks following Theorem 3, and the limiting behaviour of $\left\{\theta_{j}^{(n)}\right\}_{n=0}^{\infty}$ for an ergodic chain (Section 2), shows that

$$
\begin{aligned}
\lim _{n \rightarrow \infty} M^{(n)} & =E\left\{f\left(X_{0}\right)\right\} \sum_{j=0}^{\infty}\left[1-\sum_{i=0}^{j} \pi_{j}\right][g(j+1)-g(j)]+g(0) \\
& =E\left\{f\left(X_{0}\right)\right\} \sum_{j=0}^{\infty} \pi_{j} g(j),
\end{aligned}
$$

by Lemma 1, and this also proves the final part of the theorem.

It should be noted that the monotone behaviour of $\left\{M^{(n)}\right\}$ depends on the stochastic monotone property of $\left\{X_{n}\right\}$ and the monotone behaviour of $f(\cdot)$ and $g(\cdot)$, but reference to Theorem 4 of Daley [2], shows that the convergence of $\gamma^{(n)}$ to zero is a consequence of the uniqueness of the stationary distribution of ergodic Markov chains.

\section{Acknowledgements}

I wish to thank my supervisor, Professor B. D. Craven who has introduced me to this field. Finally, I am indebted to the referee for substantial improvements in presentation and for extensions to Theorems 1 and 2. This work has been carried out with the help of a Department of Supply cadetship.

\section{References}

[1] B. D. Craven, 'Serial dependence of a Markov process', J. Aust. Math. Soc., 5 (1965), $299-314$.

[2] D. J. Daley, 'Stochastically monotone Markov chains', $Z$. Wahr. 10 (1968), 305-317.

[3] D. J. Daley, 'The serial correlation coefficients of waiting times in a stationary single server queue.', J. Aust. Math. Soc. 8 (1968), 683-699.

[4] F. G. Foster, 'On the stochastic matrices associated with certain queueing processes', Ann. Math. Stat. 24 (1953), 355-360.

Department of Mathematics

Monash University, Melbourne 